\title{
Article
}

\section{Effect of the anti-retroviral drug, rilpivirine, on human subcutaneous adipose cells and its nutritional management using quercetin}

Behl, Shalini, Adem, Abdu, Hussain, Arif and Singh, Jaipaul

Available at http://clok.uclan.ac.uk/33177/

Behl, Shalini, Adem, Abdu, Hussain, Arif and Singh, Jaipaul ORCID: 0000-00023200-3949 (2020) Effect of the anti-retroviral drug, rilpivirine, on human subcutaneous adipose cells and its nutritional management using quercetin. Molecular and Cellular Biochemistry . ISSN 0300-8177

It is advisable to refer to the publisher's version if you intend to cite from the work. http://dx.doi.org/10.1007/s11010-020-03744-4

For more information about UCLan's research in this area go to http://www.uclan.ac.uk/researchgroups/ and search for <name of research Group>.

For information about Research generally at UCLan please go to http://www.uclan.ac.uk/research/

All outputs in CLoK are protected by Intellectual Property Rights law, including Copyright law. Copyright, IPR and Moral Rights for the works on this site are retained by the individual authors and/or other copyright owners. Terms and conditions for use of this material are defined in the policies page. 
Effect of the anti-retroviral drug, rilpivirine, on human subcutaneous adipose cells and its nutritional management using quercetin

Shalini Behl ${ }^{1 *}$, Abdu Adem ${ }^{2}$, Arif Hussain ${ }^{3}$ and Jaipaul Singh ${ }^{1}$

SBehl@uclan.ac.uk, abdu.adem@uaeu.ac.ae,dr.arifhussain@yahoo.co.in,jsingh3@uclan.ac.uk

${ }^{1}$ School of Forensic and Applied Sciences, University of Central Lancashire, Preston, England, UK, ${ }^{2}$ Department of Pharmacology, United Arab Emirates University, Al Ain, United Arab Emirates and ${ }^{3}$ School of Life Sciences, Manipal Academy of Higher Education, Dubai, United Arab Emirates.

Key words: Rilpivirine, quercetin, adipogenesis, inflammatory markers, oxidative markers.

\section{* Correspondence:}

Shalini Behl

School of Forensic and Applied Sciences,

University of Central Lancashire,

Preston PR1 2HE, Lancashire

England, United Kingdom

Email: behl.shalini@gmail.com

Mobile: 00971559346048 


\section{ABSTRACT}

Rilpivirine, a recently developed drug of choice for initial treatment of HIV-1 infection, can greatly reduce HIVrelated inflammation, but in turn, may be associated with adverse secondary effects, including disturbances in lipid metabolism and ultimately in adipose tissue distribution and function. In recent years, research findings on the benefits of anti-oxidant foods and supplements have been employed in counter-acting both oxidative stress as well as inflammation in order to reduce the adverse side effects of anti-retroviral therapy. One such natural flavonoid which possesses anti-inflammatory and anti-oxidative properties is quercetin. This study investigated the effect of quercetin in overcoming the side effects incurred due to rilpivirine administration. The results show substantial reduction in the accumulation of triglyceride levels in a dose- and time- dependent manner for adipose cells treated with either rilpivirine or quercetin alone and in combination, as evidenced by morphological pictures and quantitative measurement of triglycerides throughout the differentiation process. Levels of inflammatory markers such as resistin and IL-8 were increased as compared to the untreated cells. No significant changes in leptin were observed on treatment of adipose cells with rilpivirine alone and its levels were almost comparable to control. Levels of oxidative markers like superoxide dismutase, catalase and glutathione were also decreased. Treatment with quercetin showed a decrease in the inflammatory status and an increase in the oxidative status of adipose cells, thereby, exhibiting its anti-inflammatory and anti-oxidative properties. However, further assessment of lipid metabolism and adipose tissue function in patients administered with rilpivirine-based regimes is advisable considering that totally neutral effects of rilpivirine on lipid homeostasis cannot be anticipated from the current study in vitro. It is concluded that rilpivirine causes an antiadipogenic and pro-inflammatory response pattern but only at high concentrations, whereas quercetin has been observed to decrease inflammation and restore the levels of anti-oxidant enzymes.

\section{INTRODUCTION}

The use of antiretroviral treatment for HIV infection has radically transformed the prognosis of HIV infected patients from terminal illness to a chronic manageable condition [1,2]. Long-term treatment of HIV 1-infected patients with antiretroviral drugs may result in the appearance of several secondary effects, the most frequent being the lipo-dystrophy syndrome [3]. Lipo-dystrophy is associated with both morphological (lipo-atrophy, lipo-hypertrophy) and metabolic (dyslipidemia, glucose intolerance, hypertension, endothelial dysfunction and atherosclerosis) alterations, which may occur singly or in combination. Moreover, it is associated with an increased risk of diabetes and cardiovascular diseases (CVDs). Adipocyte inflammation, oxidative stress and 
macrophage infiltration, as well as altered adipocyte function may be considered as major contributors in the development of HIV associated lipodystrophy syndrome. The adipocyte, therefore, represents a plausible target for treatment. Although surgical treatments as well as pharmacological interventions have shown some effects, their use is associated with numerous adverse side effects and complications [4-7]. As such, lifestyle modifications may provide useful alternatives for managing the lipodystrophy syndrome, owing to their safety and tolerability.

The World Health Organization (WHO) recommends that dietary interventions should be part of all HIV/AIDS control and treatment program as it is believed that adequate nutrition can improve adherence to anti-retroviral therapy (ART) and its effectiveness. It may also help to tackle metabolic abnormalities in people living with HIV/AIDS [8]. In 1989, WHO had declared the necessity to assess ethno-medicines and other natural products for the management of HIV/AIDS. To quote: "In this context, there is need to evaluate those elements of traditional medicine, particularly medicinal plants and other natural products that might yield effective and affordable therapeutic agents. This will require a systematic approach" $[9,10]$. Moreover, since these phytomedicines are not fairly expensive and have less side effects, then they are now retrieving patient approval [11, 12]. The use of complementary and alternative medicines, including herbal medicines, is common among individuals with HIV and the reported prevalence of herbal remedies use by HIV-infected individuals in the United States varies between $12 \%$ and $27.2 \%$ [13-15]. Surveys have revealed that nearly $67 \%$ of HIV-infected patients receiving ARV therapy also take a natural health product [16]. Most common among these are vitamins and some flavanoids [17]. The major reasons reported by HIV-infected patients for using these complementary alternative medicinal modalities are a perceived additional efficacy, an increase in quality of life, a reduction of adverse effects of ARV therapy, and a feeling of control [18].

Rilpivirine (Trade name: Edurant), a prescription medicine approved by the U.S. Food and Drug Administration (FDA) is used for the treatment of HIV infection in adults and children 12 years of age and older having a viral load (number of HIV RNA copies / $\mathrm{mL}$ of blood) of 100,000 copies $/ \mathrm{mL}$ or less at the start of treatment [19]. It belongs to a class (group) of HIV drugs called non-nucleoside reverse transcriptase inhibitors (NNRTIs) and acts by binding directly to the enzyme at a site different from the nucleoside binding component, thereby causing an allosteric inhibition of the transcriptase. On the other hand, quercetin, a plant pigment, is a potent antioxidant flavonoid found mostly in onions, grapes, berries, cherries, broccoli, and citrus fruits. It is a versatile antioxidant known to possess protective abilities against tissue injury induced by various drug 
toxicities. It is also a well-recognized bioflavonoid known for its anti-inflammatory, antihypertensive, antiobesity, anti-hyper-cholesterolemic and anti-atherosclerotic activities [20, 21]. A number of cell culture studies investigating the potential anti-obesity effect of quercetin on obesity found that in addition to the inductive effect on lipolysis, quercetin can also suppress lipogenesis by reducing the incorporation rate of fatty acids into adipocyte triacylglycerols and by inhibiting the gene expression levels of fatty acid synthase (FAS) and the activity of acetyl-CoA carboxylase (ACC). Animal studies showed that quercetin can transiently increase energy expenditures which may relate to the upregulation of UCP-1. It also exhibited anti-inflammatory effects on adipose tissue and was reported to reduce the levels of inflammatory markers IFN $\gamma$, TNF $\alpha$, IL- 1 , and IL-4 in mice. It suppressed the accumulation and activation of immune cell and improves mitochondrial functions in adipose tissue of HFD-induced obese mice by increasing the levels of oxidative stress-sensitive transcription factor and antioxidant enzymes. In addition to cell culture and animal studies focused on the beneficial effects of quercetin in obesity, a limited number of human studies and clinical trials have been performed to evaluate the effects of quercetin on obesity treatment. These studies demonstrated that quercetin significantly decreased the total body fat, body mass index (BMI), waist circumference and triacylglycerol concentration. The present study reports that rilpivirine alters adipogenic differentiation, induces expression of proinflammatory cytokines and alters intracellular redox status in adipose cells. Quercetin, however, exhibits beneficial nutritional effects by overcoming the adverse effects.

\section{MATERIALS AND METHODS}

\section{Cell culture, seeding and proliferation}

To model the effect of the antiviral drug rilpivirine $(10-20 \mu \mathrm{M})$, human subcutaneous pre-adipocytes purchased from Lonza (Cat. \# PT5001) were used either alone or in the presence of an anti-inflammatory flavanoid, quercetin $(50 \mu \mathrm{M})$. Pre-warmed $\left(37^{\circ} \mathrm{C}\right)$, supplemented medium was used for the culturing of pre-adipocytes. It was prepared by adding the entire contents of the fetal bovine serum (FBS), L-Glutamine and GA-1000 SingleQuots ${ }^{\mathrm{TM}}$ to the bottle of Pre-adipocyte Basal Medium-2. The final concentrations of the supplements were $10 \%, 2 \mathrm{mM}, 30 \mu \mathrm{g} / \mathrm{ml}$ and $15 \mathrm{ng} / \mathrm{ml}$, respectively. Thawed cell suspension was added to $50 \mathrm{ml}$ of pre-warmed Pre-adipocyte Growth Medium-2 and centrifuged at $300 \mathrm{x}$ g for 10 minutes at room temperature. The cells were then washed leaving a minimum of $1 \mathrm{ml}$ of wash at the bottom of the tube. A total of $20 \mu \mathrm{l}$ of the cell suspension was finally diluted with an equal amount of $0.4 \%$ trypan blue. Cell count was done using hemocytometer to determine percent viability. 
For the expansion of cells prior to use for assays, the pre-adipocytes were plated with approximately 9,000 cells/ $\mathrm{cm}^{2}$ in $0.2 \mathrm{ml} \mathrm{medium} / \mathrm{cm}^{2}$ growth area in tissue culture flasks. The flasks were then placed in $\mathrm{CO}_{2}$ incubator (Forma $^{\mathrm{TM}}$ Series II 3110 Water-Jacketed incubator from Thermo Fisher Scientific) maintained at $37^{\circ} \mathrm{C}$ and $5 \%$ $\mathrm{CO}_{2}$. The pre-adipocytes appeared round when first plated and within four hours, greater than $90 \%$ of the cells began to flatten and elongate. Within 24-36 hours the cells were adherent, elongated and spindle shaped. As they began to further divide, they attained a rounded shape. The cultures were fed every 3-4 days after plating. For sub-culturing, the spent media were aseptically removed and discarded from the flasks followed by washing of the attached cell layer with Dulbecco's Phosphate Buffered Saline. Appropriate volume of Trypsin-EDTA solution was then added to cover the cell monolayer (approx. $25 \mathrm{ml} / \mathrm{cm}^{2}$ ). The plates were incubated at room temperature for 3 minutes and the cells were then observed under the microscope. Once $>90 \%$ of the cells were rounded and detached, approximately $0.2 \mathrm{ml} / \mathrm{cm}^{2}$ of temperature equilibrated Pre-adipocyte Growth Medium-2 was added to each flask. To remove the trypsin, the cells were centrifuged at $300 \mathrm{x} \mathrm{g}$ for 10 minutes at room temperature and the cell pellet was re-suspended in a minimal volume of temperature equilibrated growth medium. The total number of cells was counted using a haematocytometer. Differentiation of pre-adipocytes into mature adipose cells was induced by the addition of Adipocyte Differentiation Medium for a period of 8 days.

The present study investigated the effect of rilpivirine alone $(10-20 \mu \mathrm{M})$ and then in combination with quercetin $(50 \mu \mathrm{M})$. The process was initiated on day 0 and continued throughout the adipocyte differentiation period of 8 days. Stock solutions of treatment drugs were prepared in DMSO. They were then aliquoted and stored at $-20^{\circ} \mathrm{C}$ prior to use. Working solutions of the treatment drugs were prepared in pre-adipocyte growth medium-2 by diluting them further with differentiation medium.

\section{Lactate dehydrogenase (LDH) Assay for the measurement of cell cytotoxicity}

CytoTox 96 kit from Promega was used to determine the potential cytotoxic effects of the two drugs on differentiating human pre-adipocytes after exposure to cells for 15 days. The assay measured the appearance of LDH, a stable cytocyclic enzyme normally released upon cell lysis, in the cell culture medium. The released LDH in culture supernatants was measured with a 30-minute coupled enzymatic assay, which resulted in the conversion of a tetrazolium salt (iodonitro-tetrazolium violet; INT) into a red formazan product. The amount of colour formed was proportional to the number of lysed cells. Cell viability was assessed on day 3 and day 15 for 
both the drugs, alone and in combination, for all concentrations used in the study. Following experimental treatment, supernatant samples were transferred to a 96 well plate and an equal volume of CytoTox 96® Reagent was added to each well and incubated for 30 minutes. Stop Solution was added, and the absorbance signal was measured at $490 \mathrm{~nm}$ using a plate reader.

\section{Adipo-Red Staining and Quantitative measurement of triglyceride accumulation}

Visualization and quantification of intracellular lipid droplets was done using AdipoRed ${ }^{\mathrm{TM}}$ reagent following the manufacturer's protocol. AdipoRed ${ }^{\mathrm{TM}}$ stain was used to determine the effects of test compounds on the differentiation of adipocyte precursors as characterized by the accumulation of intracellular triglycerides. Cells were seeded at $10,000 / \mathrm{cm}^{2}$ of the plate surface area in appropriate volumes $(2-3 \mathrm{ml} / \mathrm{well})$ of cell culture media. Immediately prior to the assay, each plate was rinsed with PBS followed by the addition of AdipoRed ${ }^{\mathrm{TM}}$. The plate was then incubated for 10-15 minutes. The fluorescence was measured with excitation at $485 \mathrm{~nm}$ and emission at $572 \mathrm{~nm}$

\section{Quantitative Measurement of Adipokines and Cytokines}

Immuno-based human Elisa kits commercially available from R\&D Systems were used to quantitatively measure the adipocytokines (adiponectin, leptin, resistin, interleukin-8) released by adipocytes and accumulated in the cell culture medium at the end of 8 days of differentiation process. To prepare standards for all assays, each vial was reconstituted with $0.5 \mathrm{ml}$ of reagent diluent. A seven-point standard curve using 2-fold serial dilutions was prepared as described by the manufacturer.

To prepare the micro-plate for assay, the captured-antibody was diluted to the working concentration in PBS without carrier protein and a volume of $100 \mathrm{ml}$ was immediately coated onto each well. The plate was then sealed and incubated overnight at room temperature. Each well was aspirated and washed 3 times with a $400 \mu 1$ volume of wash buffer using an auto washer (HydroFlex ${ }^{\mathrm{TM}}$ micro-plate washer from Tecan). Blocking was done by adding $300 \mu \mathrm{l}$ of reagent diluent to each well. The plate was incubated at room temperature for 1 hour.

For the assay, a volume of $100 \mu \mathrm{l}$ of either sample or standards, in duplicates, were added to each of the designated wells and incubated for 2 hours at room temperature. This was followed by the addition of $100 \mu$ of the detection antibody. A volume of $100 \mu$ of the working dilution (1:200) of Streptavidin-HRP was added to each well and incubated for 20 minutes at room temperature. Thereafter, aspirate/wash steps were repeated. A volume of $100 \mu \mathrm{l}$ of substrate solution was added to each well and incubation was done at room temperature for 
20 minutes. Any exposure to direct light was avoided. A volume of $50 \mu$ of stop solution was added to each well and optical density was determined immediately, using a micro plate reader (Molecular Devices from Life technologies ${ }^{\mathrm{TM}}$ equipped with Softmax pro7 Version 4.3 software) set to $450 \mathrm{~nm}$.

For obtaining the results, average zero standard optical density was subtracted from mean absorbance of each set of duplicate standards, controls and samples. Standard curve was prepared by plotting the mean absorbance for each standard on the $\mathrm{y}$-axis against the concentration on the $\mathrm{x}$-axis and drawing a best fit curve through the points on the graph. Sample concentrations were calculated using the equation of the standard curve.

\section{Quantitative measurement of oxidative stress markers}

Markers of oxidative stress (Superoxide dismutase, Glutathione, Nitric oxide and Catalase) were quantified using the cell lysates from the cultures using commercially available kits.

\section{Superoxide Dismutase (SOD) levels}

Superoxide Dismutase Assay kit (Cat. \# 706002) purchased from Cayman chemicals was used to determine the SOD levels. Standard and sample wells were prepared by adding $200 \mu$ of the diluted radical detector and $10 \mu 1$ of SOD standard or sample per well. The reaction was initiated by adding $20 \mu$ of diluted xanthine oxidase to all the wells. The plate was then kept on a shaker for 30 minutes at room temperature and absorbance was read at 440-460 nm using a plate reader. Average absorbance for each standard and sample was calculated by subtracting the sample background absorbance from the sample. Linearized rate was obtained by dividing standard A's absorbance by itself and then with absorbance values of all other standards and samples. This linearized SOD standard rate was plotted as a function of final SOD Activity. SOD activity was calculated by using the equation obtained from the linear regression of the standard curve and substituting the linearized rate for each sample.

\section{Glutathione Assay}

This assay was performed using the Glutathione Assay Kit from Sigma-Aldrich (Cat. \#CS0260) following the manufacture's protocol. Standards were prepared by adding $50 \mu \mathrm{M}$ of glutathione solution in the first well and then subsequently diluting it serially. In order to prepare the samples for the assay, cell extracts were washed with PBS and at least $1 \times 10^{8}$ cells were suspended per ml of PBS and transferred to a micro centrifuge tube. Centrigugation was done at $600 \mathrm{x}$ g and supernatant was removed. The volume of the pellet was measured and 3 
volumes of 5\% SSA solution was added. The suspension was then freeze-thawed twice using liquid nitrogen and a $37^{\circ} \mathrm{C}$ water bath. It was then left for 5 minutes at $2-8{ }^{\circ} \mathrm{C}$. The extract was centrifuged at $10,000 \times \mathrm{g}$ for 10 minutes and the volume of the supernatant was measured. For the assay procedure, plate reader was set to 412 $\mathrm{nm}$ with kinetic read at 1 -minute intervals for 5 minutes. The first 2 wells contained only $10 \mu 1$ of the $5 \% 5$ sulfosalicylic acid solution as a reagent blank. A total of $10 \mu \mathrm{l}$ glutathione standard solutions and unknown samples in duplicate were added into designated wells of the plate. A volume of $150 \mu$ l of working mixture was added to each well followed by 5 minutes of incubation at room temperature. After incubation, $50 \mu 1$ of diluted NADPH solution was added to each well and absorbance was measured. To compute the results, values from the glutathione standard solutions were used to determine the standard curve and calculate $\Delta \mathrm{A}_{412} /$ min equivalent to 1 mole of reduced glutathione per well.

\section{Nitric Oxide Assay}

Cayman's Nitrate/Nitrite colorimetric Assay kit (Cat. \#780001) was used for the measurement of total nitric oxide following the manufacturer's protocol. A volume of $200 \mu 1$ of assay buffer (to be used as blank) and $80 \mu 1$ of sample was added to the wells. This was followed by the addition of $10 \mu 1$ of the Enzyme Cofactor Mixture and $10 \mu \mathrm{l}$ of the nitrate reductase mixture to each of the wells (standards and unknowns). The plate was covered and incubated for 2 hours at room temperature. After the incubation time, a volume of $50 \mu 1$ of Griess Reagent $\mathrm{R} 1$ followed by $50 \mu 1$ of Griess Reagent R2 was added to each of the wells. The plate was left for 10 minutes at room temperature for the development of colour. Absorbance was read at $540 \mathrm{~nm}$ using a plate reader. Mean absorbance values of the blank wells were subtracted from the absorbance values of all the other wells. Corrected absorbance values at $540 \mathrm{~nm}$ were plotted as a function of nitric oxide concentration.

\section{Catalase Activity}

Cayman's Catalase Kit (Cat. \# 707002) was used to determine the enzyme activity of catalase using the cell lysate. Formaldehyde standard wells were prepared by adding a volume of $100 \mu$ of diluted assay buffer of standard tubes in each of the designated wells on the plate. Sample wells were prepared by addition of $100 \mu$ l of diluted assay buffer, $30 \mu \mathrm{l}$ of methanol, and $20 \mu \mathrm{l}$ of sample. Reaction was initiated by adding $20 \mu \mathrm{l}$ of diluted hydrogen peroxide to all the wells. The plate was covered and incubated on a shaker for 20 minutes at room temperature. The reaction was terminated by adding $30 \mu \mathrm{l}$ of potassium hydroxide followed by addition of $30 \mu \mathrm{l}$ of catalase purpald (Chromogen) to each well. Incubation was done for 10 minutes at room temperature on the 
shaker. After this, a volume of $10 \mu \mathrm{l}$ of catalase potassium periodate was added to each well and incubation was done for 5 minutes at room temperature. Absorbance was read at $540 \mathrm{~nm}$ using a plate reader. The rate of the reaction was determined by subtracting the average absorbance of each standard and sample from the average absorbance of standard A. The corrected absorbance of standards was plotted as a function of final formaldehyde concentration $(\mu \mathrm{M})$. Formaldehyde concentration of the samples was calculated using the equation obtained from the linear regression of the standard curve after substituting corrected absorbance values for each sample.

\section{Statistical analysis}

Data were analyzed statistically using SPSS Version 25 software. The means of the data are presented with the standard deviation (SD). The results were analyzed using one-way ANOVA to determine the significance of the mean between the groups. Values of $\mathrm{p}<0.05$ were considered significant.

\section{RESULTS}

\section{Cyto-toxicity measurement using lactate dehydrogenase assay}

The human subcutaneous pre-adipocytes were seeded at a density of $2 \times 10^{4}$ cells per well in 24-well plates and cultured until $90 \%$ confluence. They were then treated with various concentrations (10-20 $\mu \mathrm{M})$ of rilpivirine or quercetin $(50 \mu \mathrm{M})$ either alone or in combination for a period of 15 days. As an indicator of cytotoxicity, the cytoplasmic enzyme lactate dehydrogenase (LDH) was quantified in the culture supernatant of the control as well as treated differentiating adipose cells on day 3 and day 15 of culture. The percentage of viable cells was calculated by defining the cell viability without treatment as $100 \%$. The results are shown in Figure 1 for (A) 3 days and (B) 15 days. The results presented in figure I a show no significant ( $p>0.05$ ) changes in the viability of cells when treated with various concentrations of either rilpivirine or quercetin either alone or in combination for day 3 of culture. The results presented in figure I $\mathrm{b}$ show that rilpivirine at lower concentrations $(10 \mu \mathrm{M}$ and $15 \mu \mathrm{M})$ and quercetin at $50 \mu \mathrm{M}$ did not alter the viability rate of the cells. However, at a concentration of $20 \mu \mathrm{M}$ rilpivirine, a slight and significant $(\mathrm{p}<0.05)$ decrease in the cell viability was observed indicating its effect at higher doses. A combination of rilpivirine and quercetin at concentrations of $20 \mu \mathrm{M}$ and $50 \mu \mathrm{M}$, respectively, showed a significant $(\mathrm{p}<0.05)$ decrease in cell viability. 


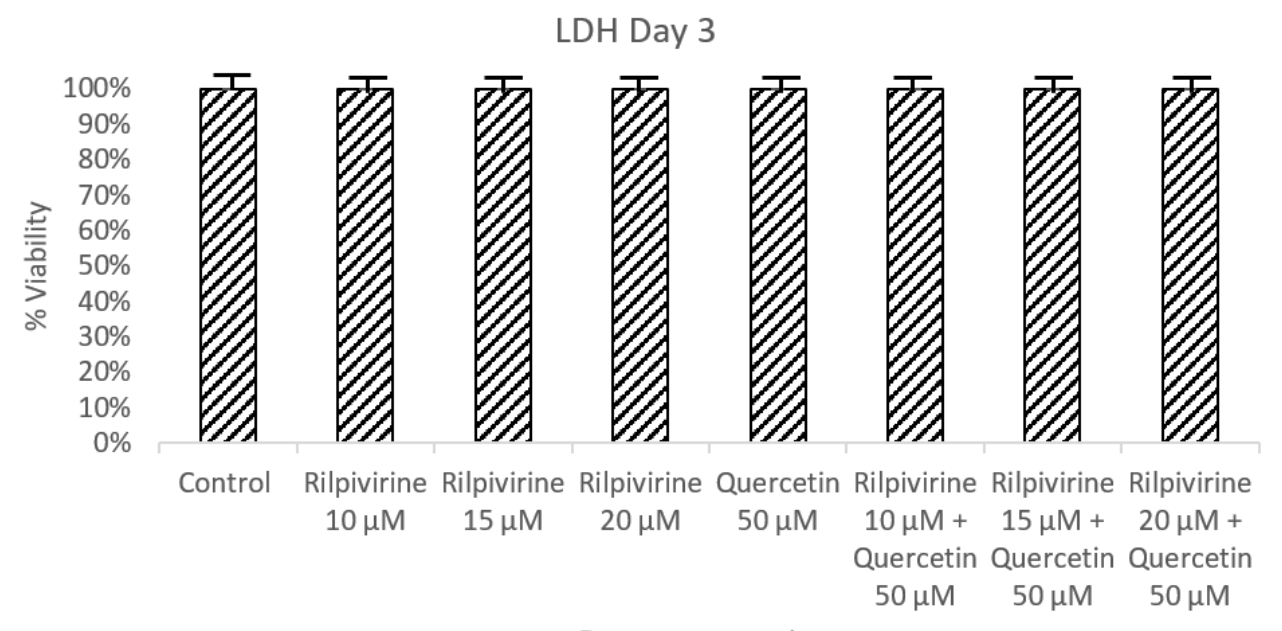

Drug concentrations

b

\section{LDH Day 15}

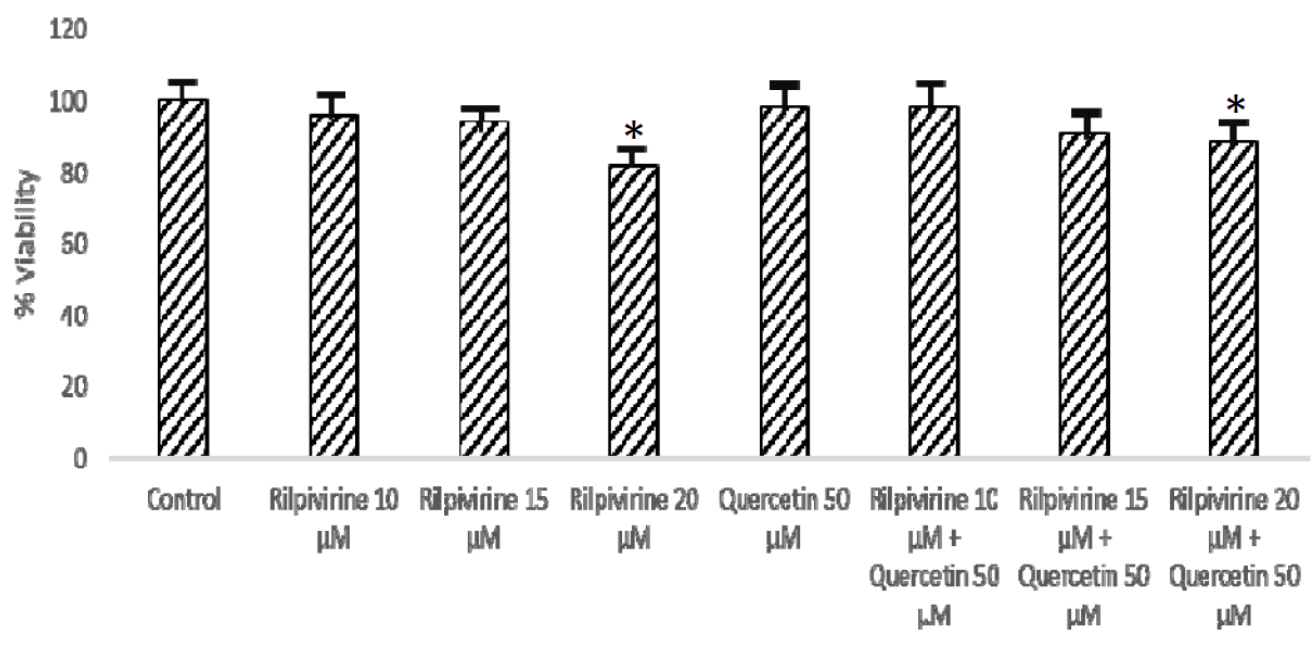

Drug concentrations

Fig I Bar graph showing the effect of either rilpivirine or quercetin either alone or in combination on the cell viability as measured by LDH on day 3 and day 15 of culture

Human subcutaneous adipose cells were treated with various concentrations of indicated drugs either alone and in combination. Cyto-toxicity was expressed as the 

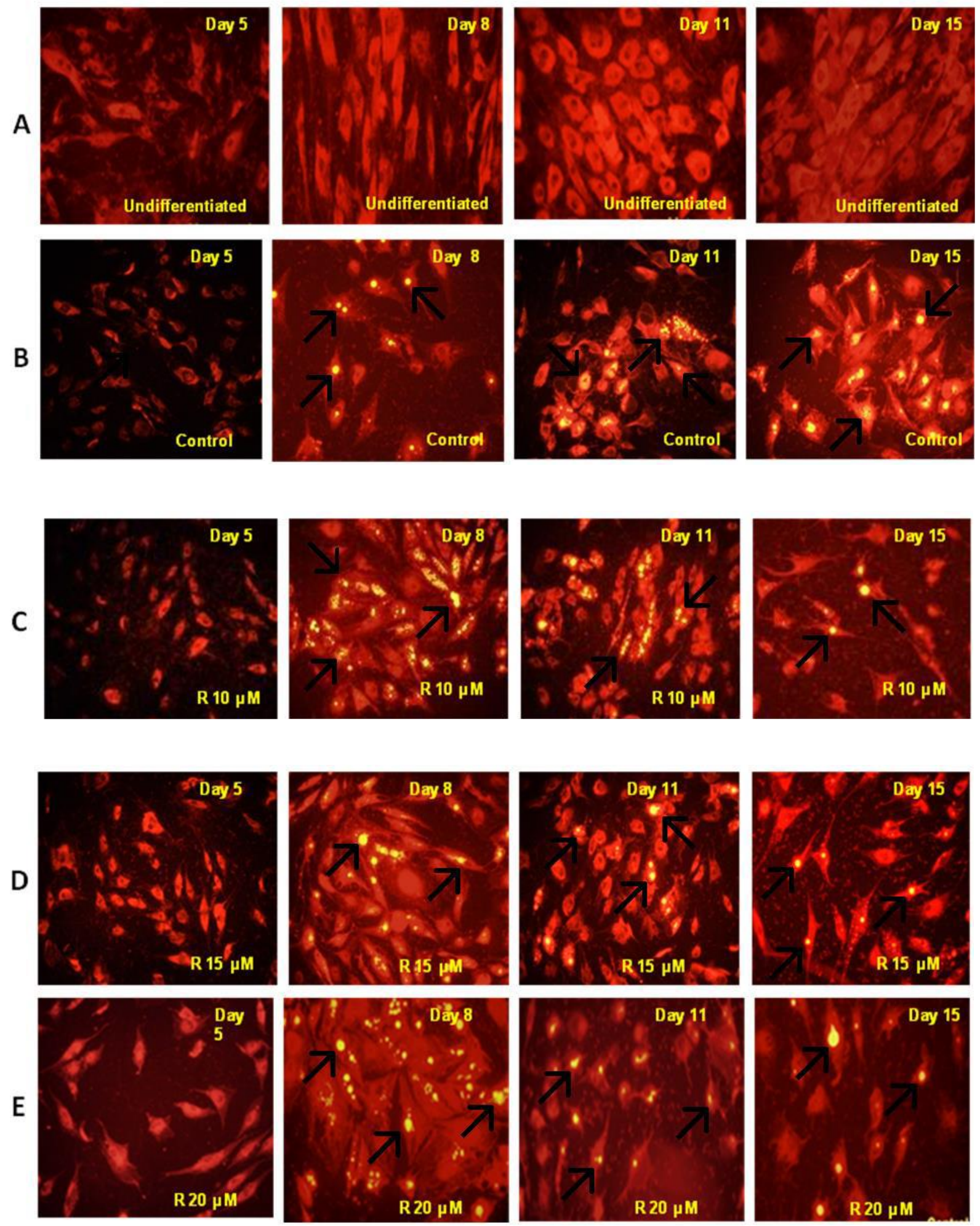

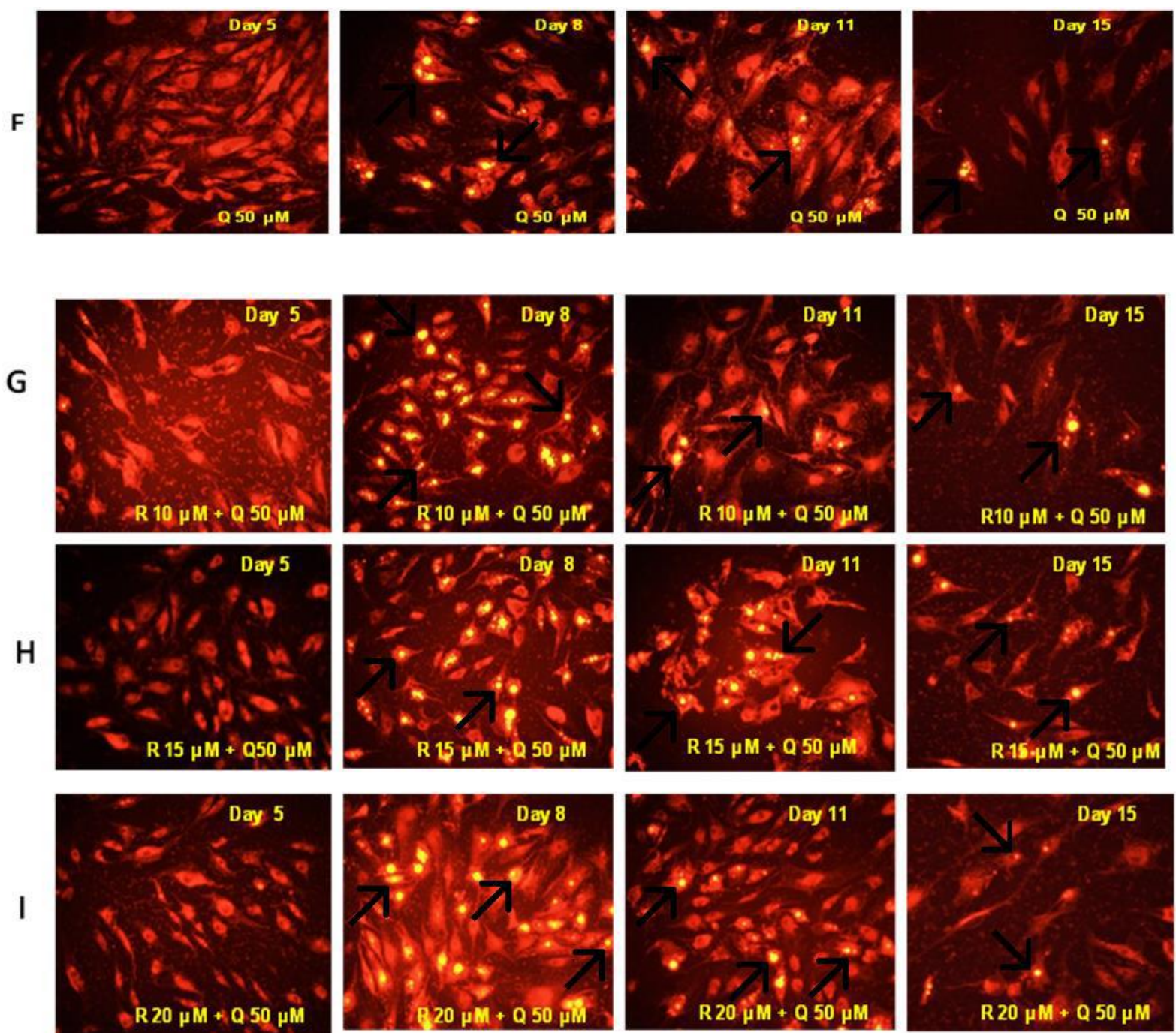

Fig II Representative images (A-I) showing the progression of adipogenesis as indicated by lipid formation for human adipose cells in culture

Human subcutaneous adipose cells were differentiated and treated with different concentrations of either rilpivirine or quercetin individually and then in combination.

Images were taken for the 5th, 8 th, 11 th and 15 th day of cells in culture at 20x magnification using a fluorescence microscope. Staining was done using adipored to visualize lipid content. Images are typical of 3 such different experiments in duplicate. These images reveal the following: A). Undifferentiated and untreated (no drugs) adipose cells. B). Differentiated and untreated (no drugs) adipose cells. C). Adipocytes differentiated and treated with $10 \mu \mathrm{M}$ of rilpivirine. D). Adipocytes differentiated and treated with $15 \mu \mathrm{M}$ of rilpivirine. E). Adipocytes differentiated and treated with $20 \mu \mathrm{M}$ of rilpivirine. F). Adipocytes differentiated and treated with $50 \mu \mathrm{M}$ of quercetin. G). Adipocytes were differentiated and treated with a combination of $10 \mu \mathrm{M}$ rilpivirine and $50 \mu \mathrm{M}$ quercetin. $\mathrm{H}$ ). Adipocytes differentiated and treated with a combination of $15 \mu \mathrm{M}$ rilpivirine and $50 \mu \mathrm{M}$ quercetin.I). Adipocytes were differentiated and treated with a combination of $20 \mu \mathrm{M}$ rilpivirine and $50 \mu \mathrm{M}$ quercetin.

Morphological fluorescence data in figure II show the accumulation of triglyceride in pre-adipocytes cultured and differentiated in the presence and absence of treatment drugs either alone or in combination. Image A 
represents undifferentiated and untreated pre-adipocyte cells in culture. These cells appeared elongated, fibroblast-like structure, spindle-shaped and showed no lipid accumulation. Image B represents the progression of pre-adipocyte cells into matured adipose cells differentiated for 15 days without any drug treatment (control). The process of adipogenesis progressed in a time-dependent manner as evidenced by the increasing lipid droplets (yellow). On day 5, almost none or very little amount of lipid droplets were observed. However, by day 8 the cells began to attain a rounder shape and became laden with lipids. They had accumulated substantial triglyceride content and grew more in size and number on day 11 and 15 of culture. Images C, D and E represent pre-adipocyte cells treated with a dosage concentration of $10 \mu \mathrm{M}, 15 \mu \mathrm{M}$ and $20 \mu \mathrm{M}$ of rilpivirine, respectively over a period of 15 days. It was observed that rilpivirine inhibited the accumulation of triglycerides in a doseand time- dependent manner. The formation of lipid droplets (yellow) decreased with increasing dosage and time in culture. Images F represents pre-adipocytes treated with $50 \mu \mathrm{M}$ of quercetin. Pre-adipocytes treated with quercetin were shown to inhibit triglyceride accumulation with increasing number of days in culture. The number of lipid droplets decreased from day 8 until day 15 in culture. Images G, H and I represent pre-adipocyte cells treated with a combination of $10 \mu \mathrm{M}, 15 \mu \mathrm{M}, 20 \mu \mathrm{M}$ of estradiol and $50 \mu \mathrm{M}$ of quercetin, respectively. Adipose cells treated with these combinations of drugs showed attenuation in lipid content in time- and dosedependent manner. Day 8 of differentiation showed a substantial amount of triglyceride accumulation which almost became diminished until day 15 .

\section{Quantitative measurement of triglycerides}

Triglycerides accumulated over the differentiation period of 15 days with drug treatments were measured quantitatively compared to untreated cells by staining the cells with adipored reagent and the fluorescence was measured at OD value of $572 \mathrm{~nm}$ and the data shown in Figure III A-D for comparison. 

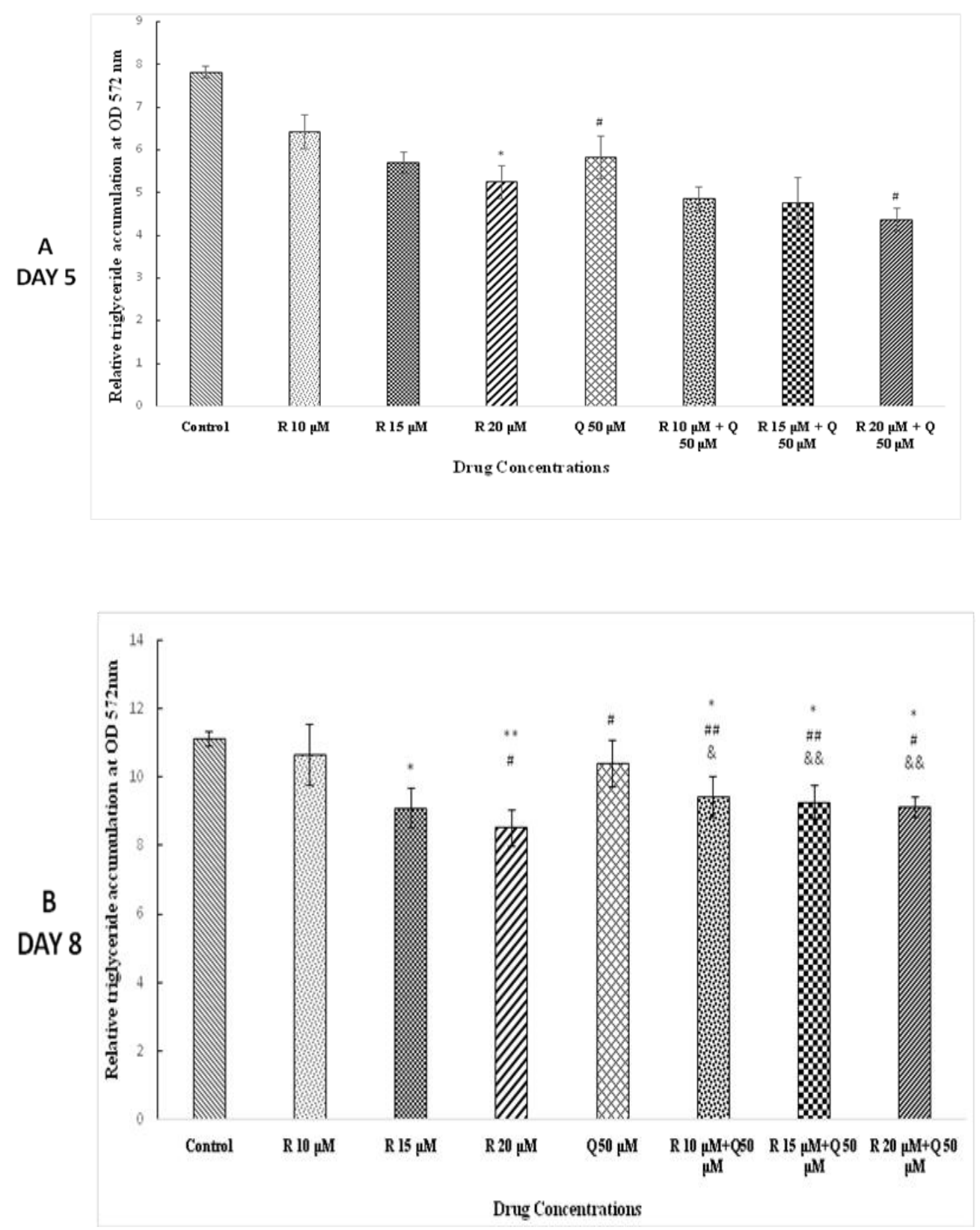

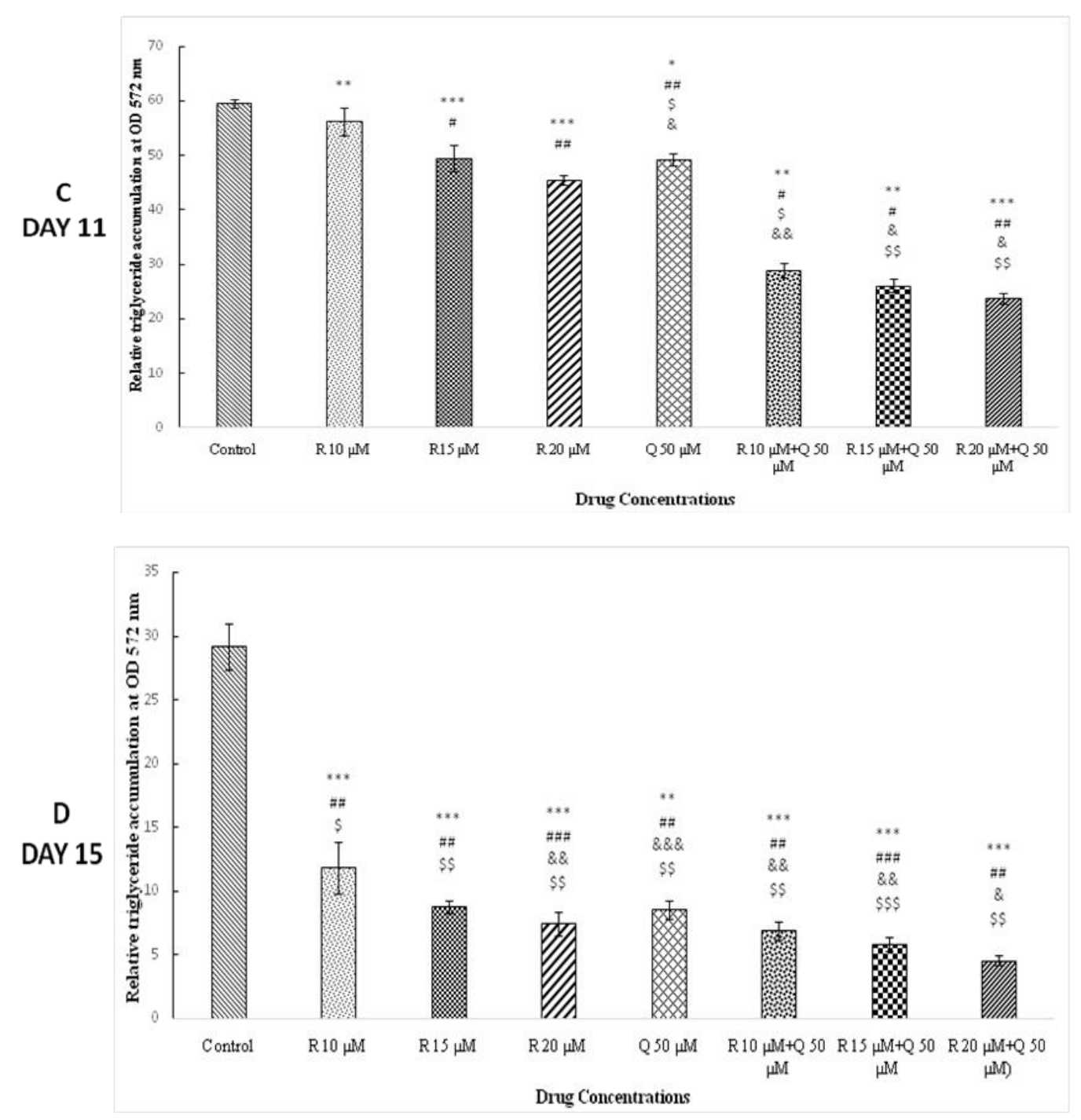

Fig III (A-D) Bar graphs showing the effect of rilpivirine and quercetin individually and then in combination on the accumulation of triglyceride by human adipocytes in culture

Human pre-adipocytes were differentiated in culture in the presence of indicated concentrations of rilpivirine and quercetin. Values represent concentration of triglyceride in cell supernatant for the 5th, 8th and 11th and 15th day of culture. Statistical analysis was performed using a one-way ANOVA and are presented as mean \pm S.D from triplicate set of experiments expressed relative to values from untreated control cells; ${ }^{*} \mathrm{p}<0.05 * * \mathrm{p}<0.01 * * * \mathrm{p}<0.001$ compared to the control. \#, \$, and indicate significance differences within the treatment groups.

Figure III A-D shows triglyceride accumulation in differentiated mature adipose cells over a period of 15 days either in the absence (control) or presence of indicated concentrations of treatment rilpivirine or quercetin, alone or in combination. Adipose cells that were not treated with any drugs (control) showed a significant progression in the accumulation of triglycerides over 15 days in culture. Figure III A shows the accumulation of 
triglycerides on the 5th day of culture. Rilpivirine at all tested concentrations induced significant $(\mathrm{p}<0.05)$ dosedependent changes in the lipid accumulation with maximum decrease at $20 \mu \mathrm{M}$ of rilpivirine. Adipose cells treated with $50 \mu \mathrm{M}$ of quercetin also evoked a slight reduction in the triglyceride levels. Combination of rilpivirine and quercetin decreased the triglyceride concentration in a dose dependent manner.

The results of triglyceride accumulation were more pronounced on day 8 and day 11 of culture (see Figure III $\mathrm{B} / \mathrm{C}$ ). Rilpivirine at concentrations of $15 \mu \mathrm{M}$ and $20 \mu \mathrm{M}$ showed a significant ( $\mathrm{p}<0.05$ and $\mathrm{p}<0.01$ for day 8 and $\mathrm{p}<0.01$ and $\mathrm{p}<0.001$ for day 11 , respectively) decrease in triglyceride levels. Quercetin also inhibited the triglyceride level significantly $(\mathrm{p}<0.001)$. When the two drugs were combined, significant reduction in triglyceride levels were measured. The levels of triglyceride on day 15 (Figure III D) in the adipose cells showed substantial reduction $(\mathrm{p}<0.001)$ when treated with either rilpivirine or quercetin. This reduction was more prominent and significant when the two drugs were combined together.

Overall, the results show that both rilpivirine and quercetin alone and in combination can significantly inhibit ( $<<0.05$ for day 8 and $\mathrm{p}<0.001$ for day 11 and 15 , respectively) lipid formation and attenuate triglyceride accumulation in adipose cells in both dose as well as time dependent manner.

Based on the results presented in figures II and III, the median dosage concentration at which rilpivirine inhibited triglyceride accumulation maximally was $15 \mu \mathrm{M}$. Thus, this concentration was chosen for a differentiation period of 8 days with $50 \mu \mathrm{M}$ quercetin measuring a number of adipocytokines and oxidative stress markers using biochemical assays.

\section{Quantitative measurement of inflammatory adipocytokines}

Human subcutaneous adipose cells were cultured and differentiated over a period of 8 days in the presence of either rilpivirine or quercetin alone and in combination using maximal dose of each drug. Supernatant was collected on the $8^{\text {th }}$ day of culture and the released adipocytokines (adiponectin, leptin, resistin, IL-8) were measured quantitatively. The data are presented in figure IV A-D.

\section{A Quantitative measurement of adiponectin}




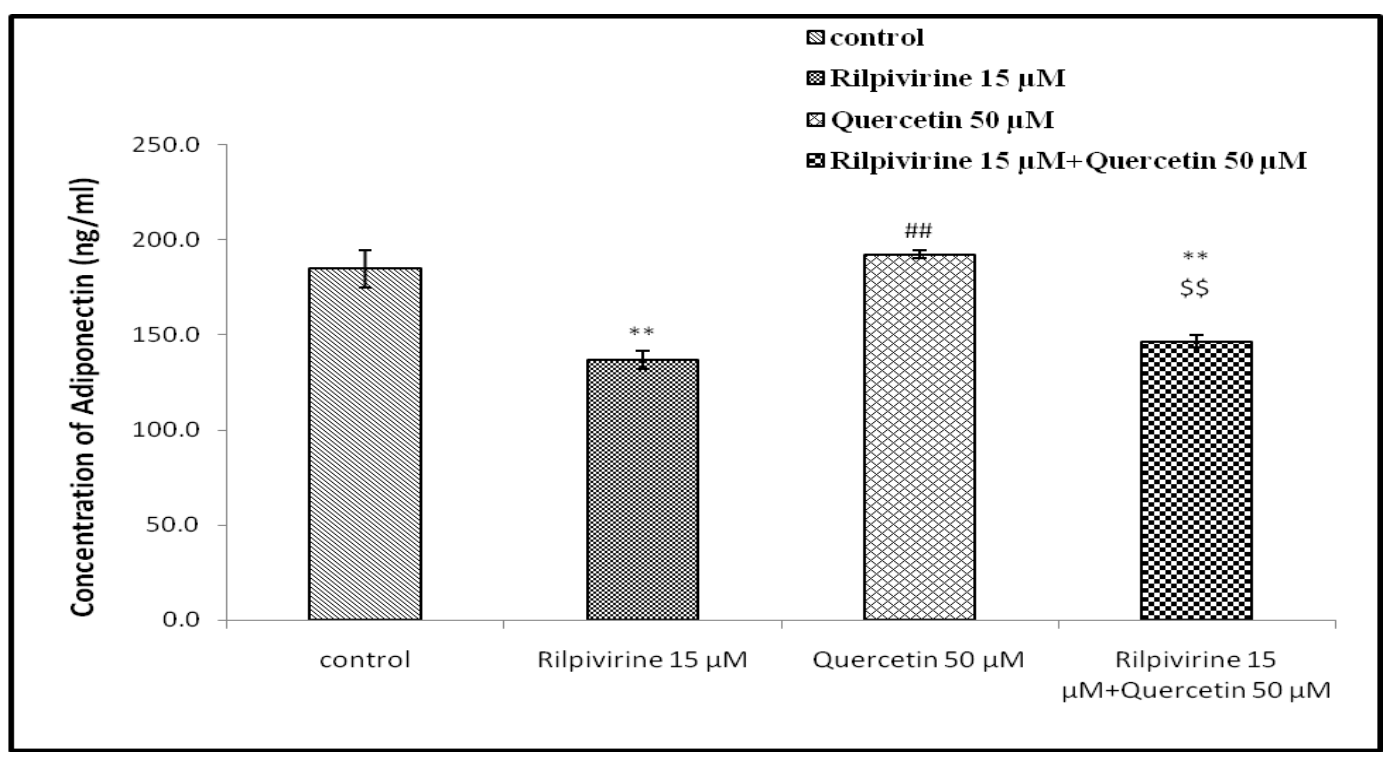

B Quantitative measurement of leptin

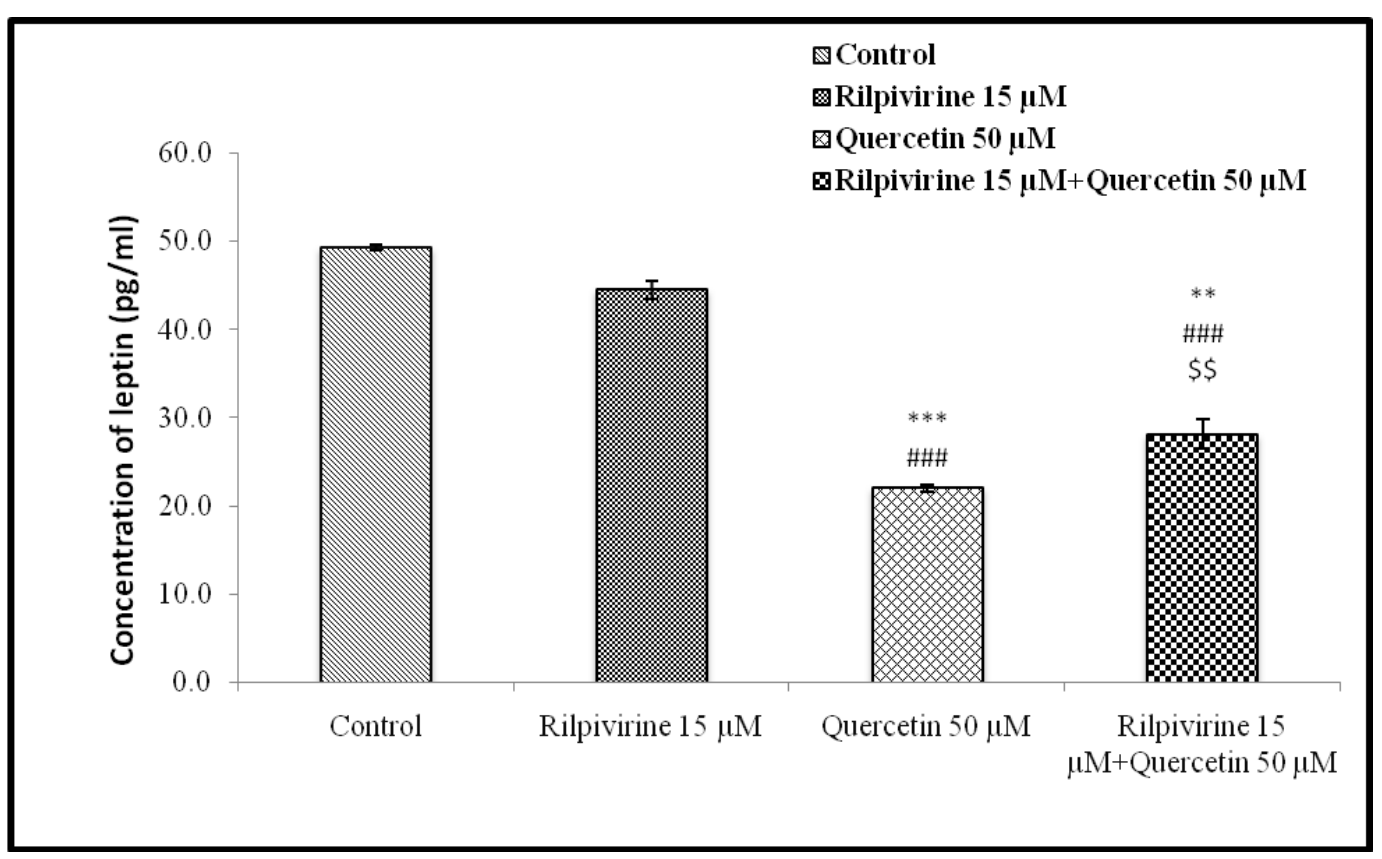

C Quantitative measurement of resistin 


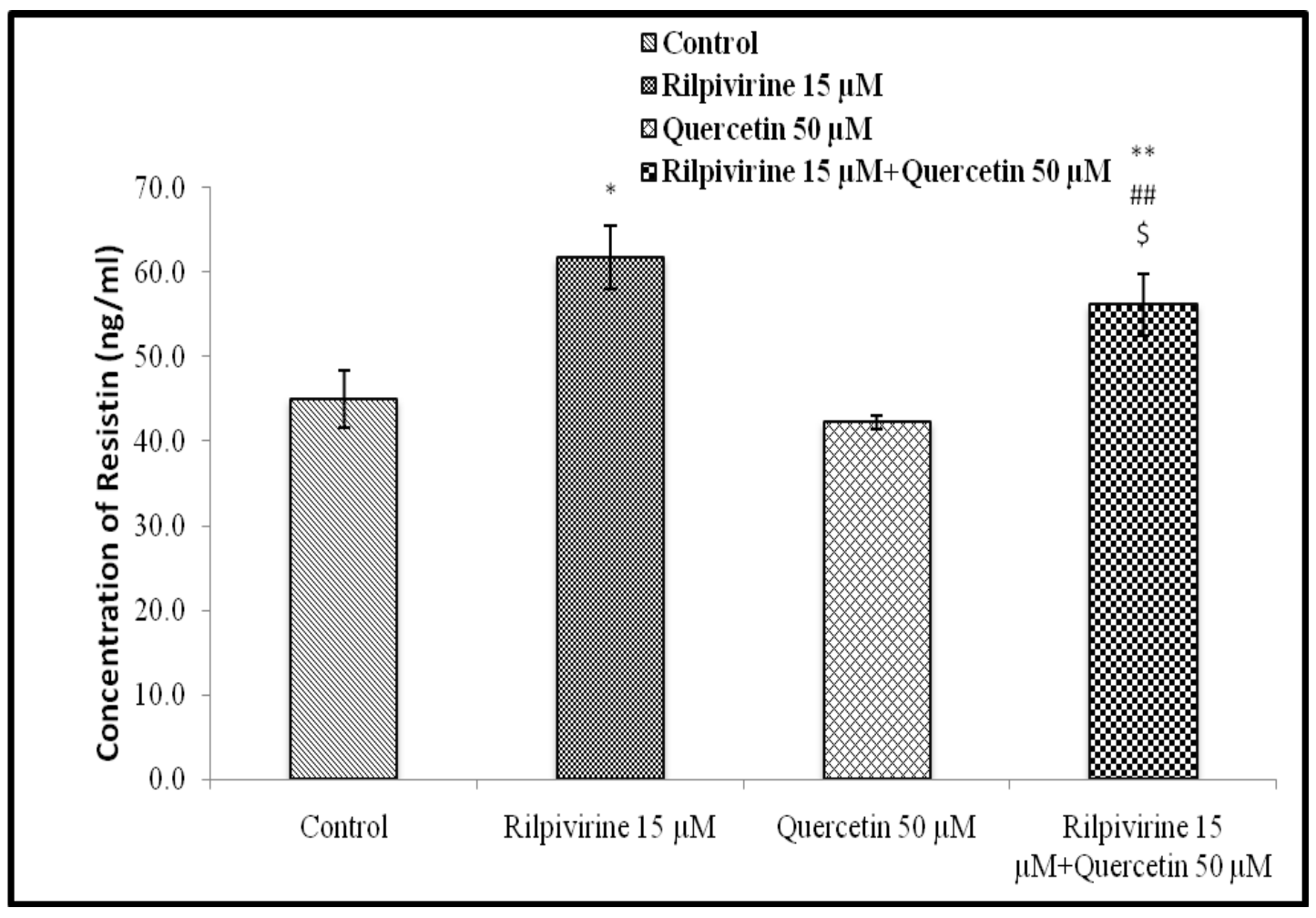

D Quantitative measurement of IL-8

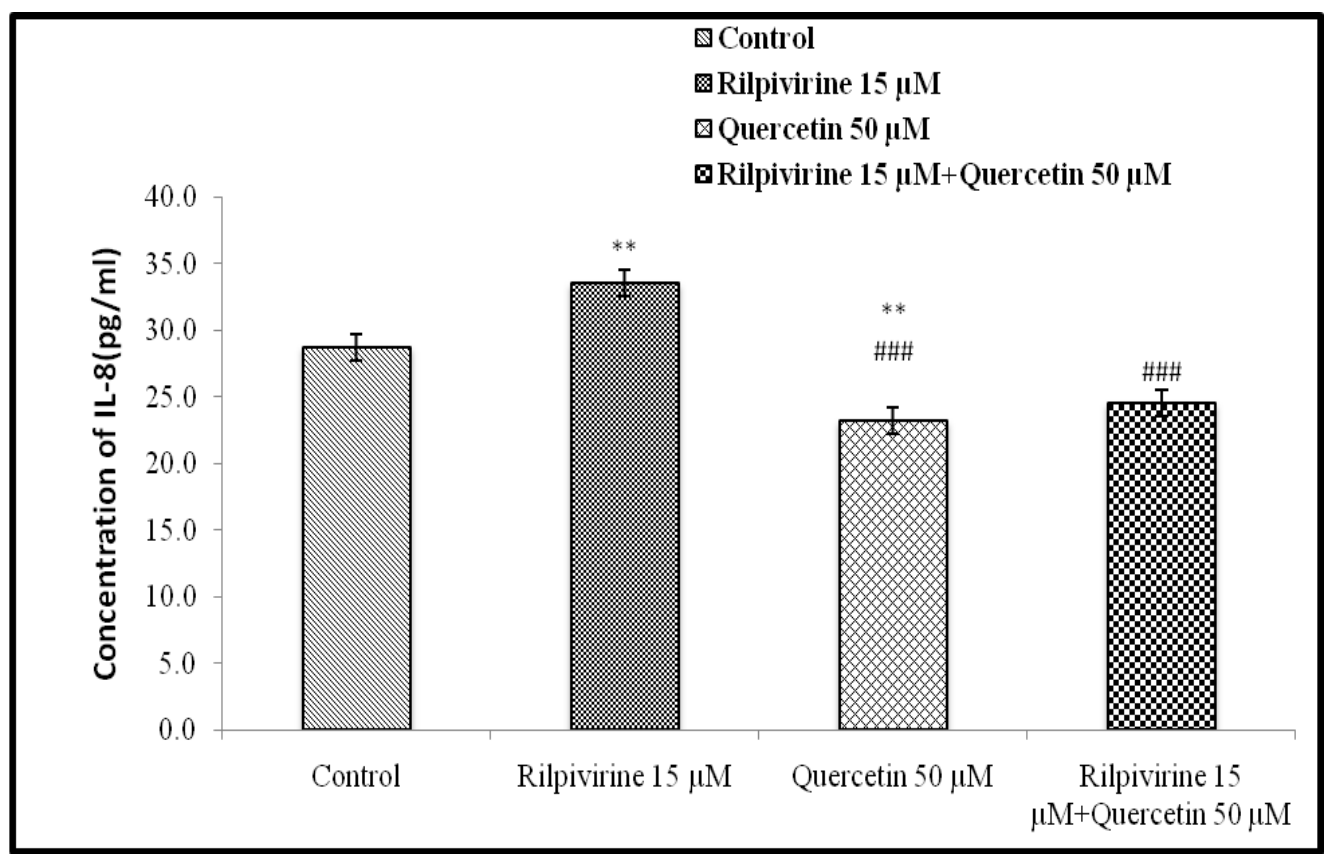


Fig IV (A-D) Bar graphs showing the effect of rilpivirine and quercetin either alone or in combination on the release of adiponectin (A), leptin (B), resistin (C) and interleukin-8 (D) by human adipocytes in culture.

Human pre-adipocytes were differentiated in the presence of indicated concentrations of drugs ( $15 \mu \mathrm{M}$ rilpivirine and $50 \mu \mathrm{M}$ quercetin). Values represent concentrations of released inflammatory markers in the cell supernatant for the 8th day of culture. Statistical analysis was performed using a one-way ANOVA and are presented as mean \pm S.D from triplicate set of experiments expressed relative to values from untreated control cells; ${ }^{* *} \mathrm{p}<0.01 * * * \mathrm{p}<0.001$ compared to the control. \#, $\$$ indicate significant differences within the treatment groups.

The results in figure IV A show marked significant reduction in the adiponectin concentration levels as compared to control when human pre-adipocytes were treated with rilpivirine alone $(\mathrm{p}<0.01)$. Significant differences were observed in the adipocytes treated with a combination of rilpivirine and quercetin when compared with control $(\mathrm{p}<0.01)$ as well as with quercetin alone $(\mathrm{p}<0.01)$. Quercetin alone showed a significant $(p<0.01)$ increase in adiponectin levels when compared to the untreated or control group. The results in figure IV B show small decrease in leptin level in the adipocytes treated with rilpivirine alone compared to control. Treatment with quercetin alone showed significant $(\mathrm{p}<0.001)$ decrease in leptin levels when compared to either control or rilpivirine alone. Adipose cells treated with a combination of rilpivirine and quercetin showed significant $(\mathrm{p}<0.01)$ decrease in the leptin levels as compared to control. The data in figure IV C show significant increase in resistin levels from adipose cells when treated with rilpivirine alone $(\mathrm{p}<0.05)$ or in combination with quercetin $(\mathrm{p}<0.01)$ as compared to control. The results also show that $50 \mu \mathrm{M}$ of quercetin had no significant effect on the release of resistin compared to control. The results in figure IV D show a significant increase in the levels of IL-8 for adipose cells treated with rilpivirine $(\mathrm{p}<0.01)$ as compared to control. Treatment with quercetin alone showed a small but significant $(\mathrm{p}<0.01)$ reduction in IL-8 levels. More or less, the same significant reduction in IL-8 concentration in the cell supernatant was obtained when the two drugs were combined.

\section{Quantitative measurement of oxidative stress markers}

Markers of oxidative stress (nitric oxide, superoxide dismutase, glutathione and catalase) were measured quantitatively using commercially available kits and the data are presented in figure V A-D for drug treated compared to untreated cells.

\section{A Quantitative measurement of catalase activity}




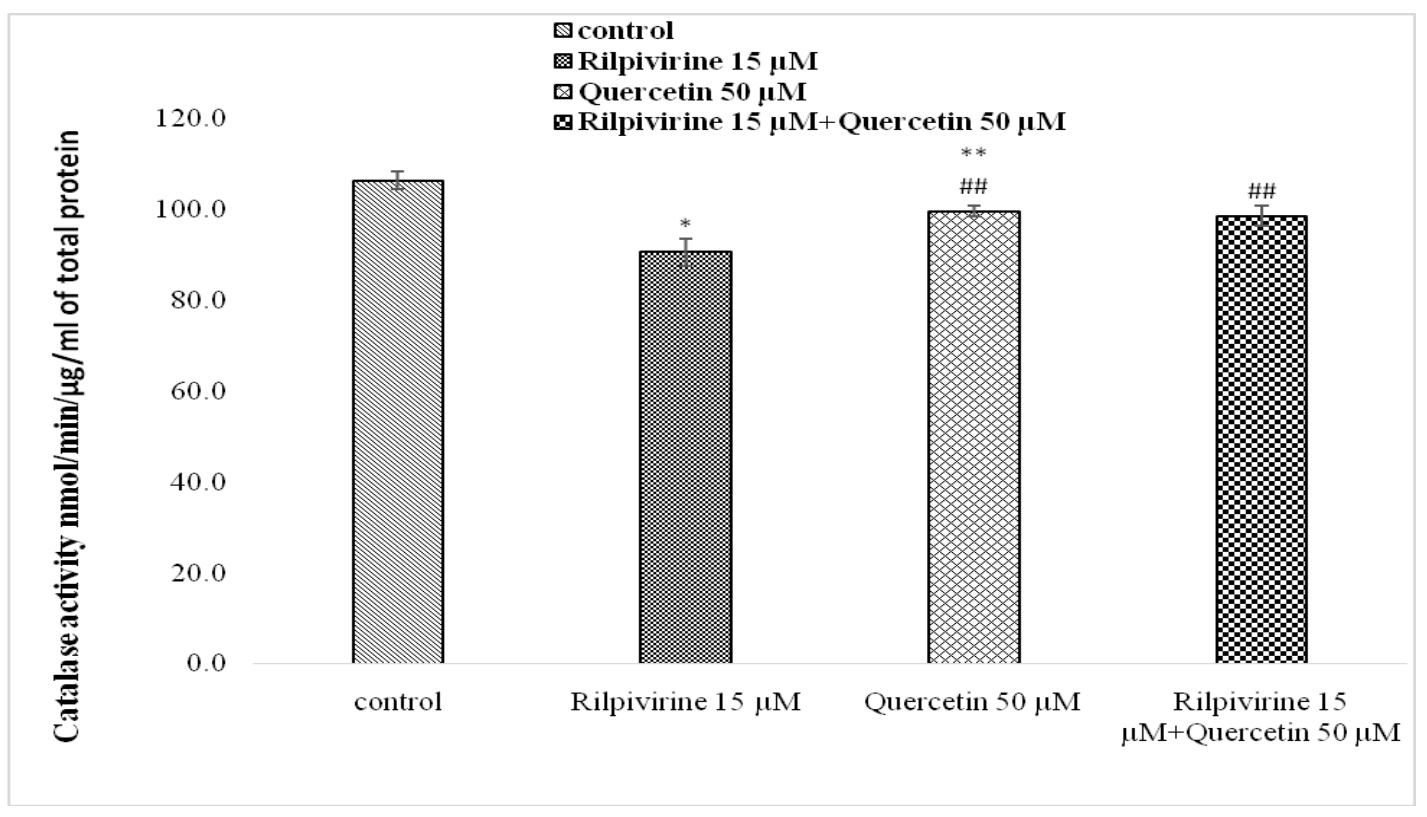

B Quantitative measurement of SOD activity

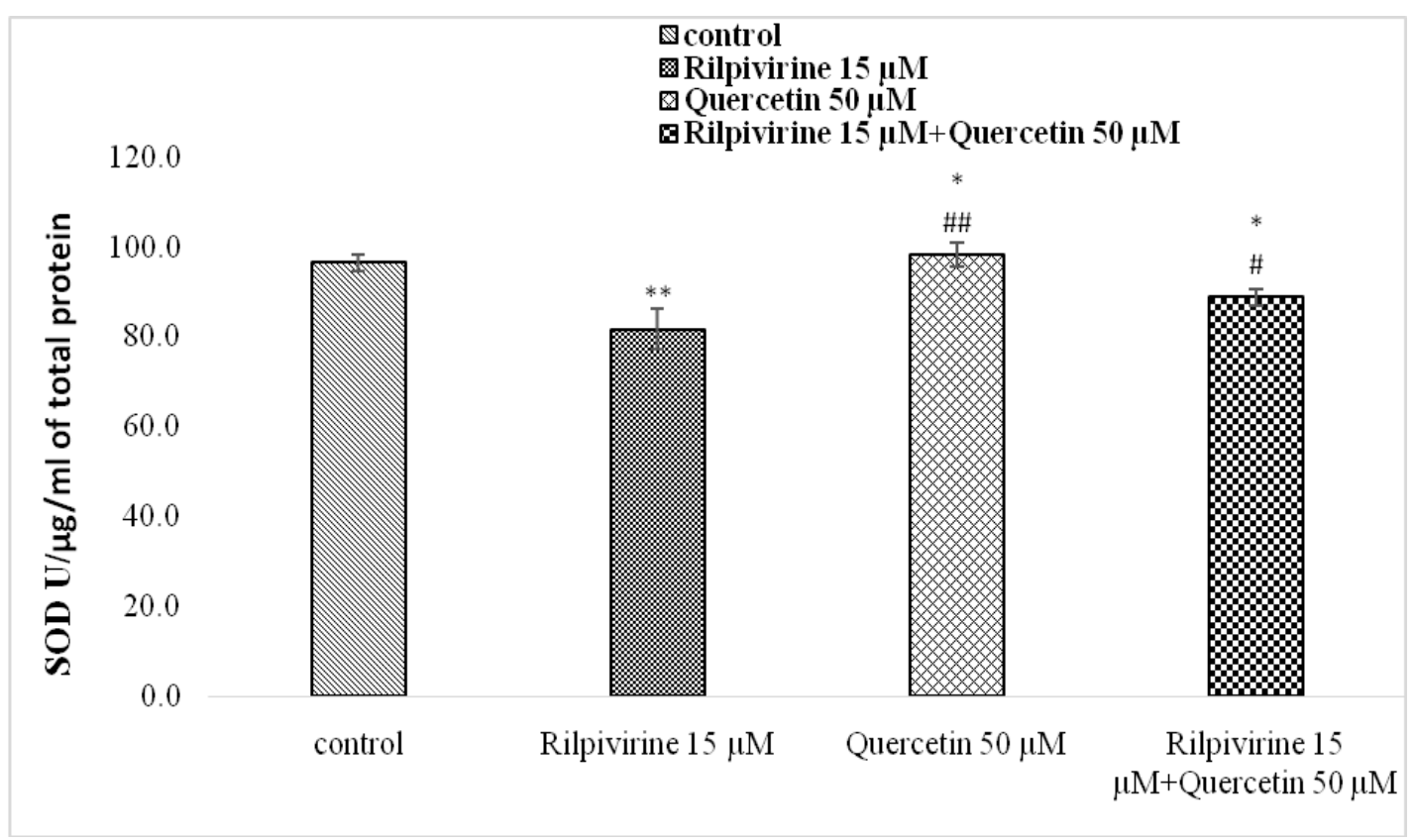

C Quantitative measurement of nitric oxide 


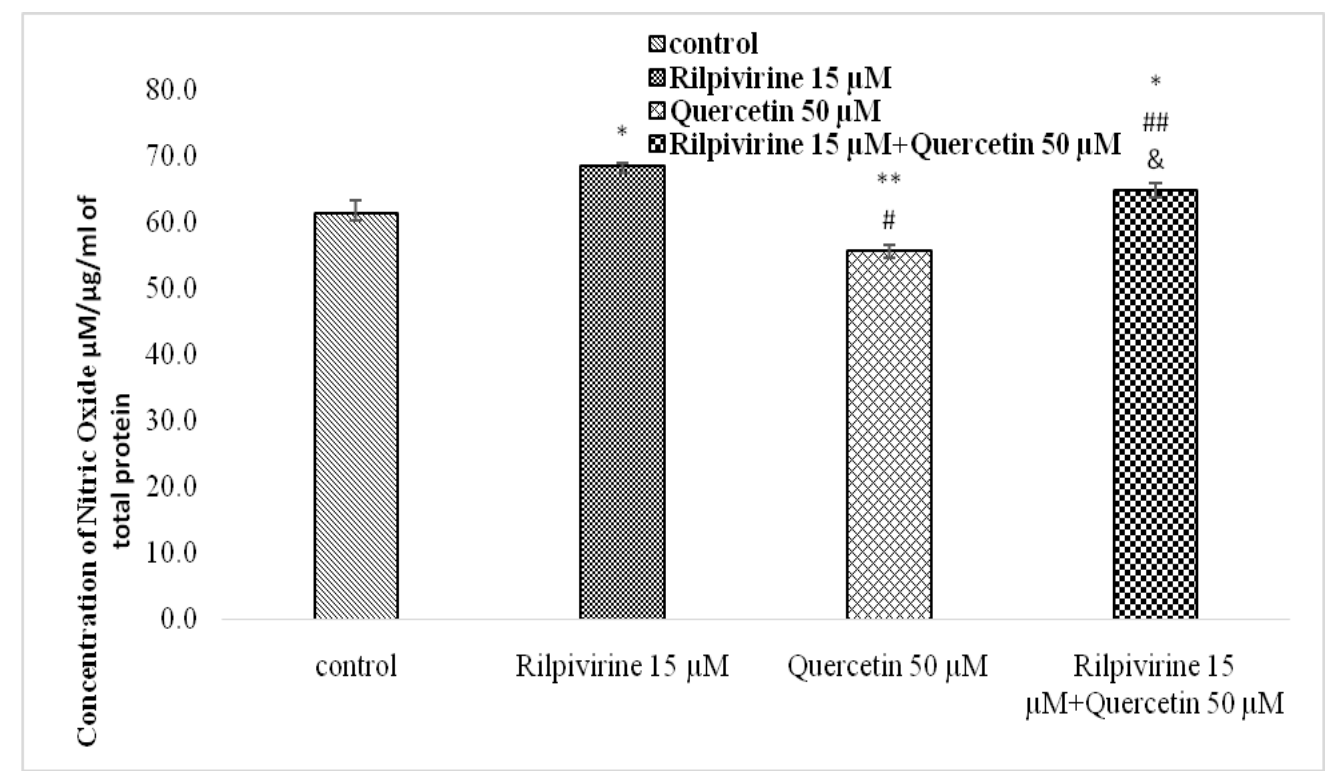

\section{Quantitative measurement of glutathione}

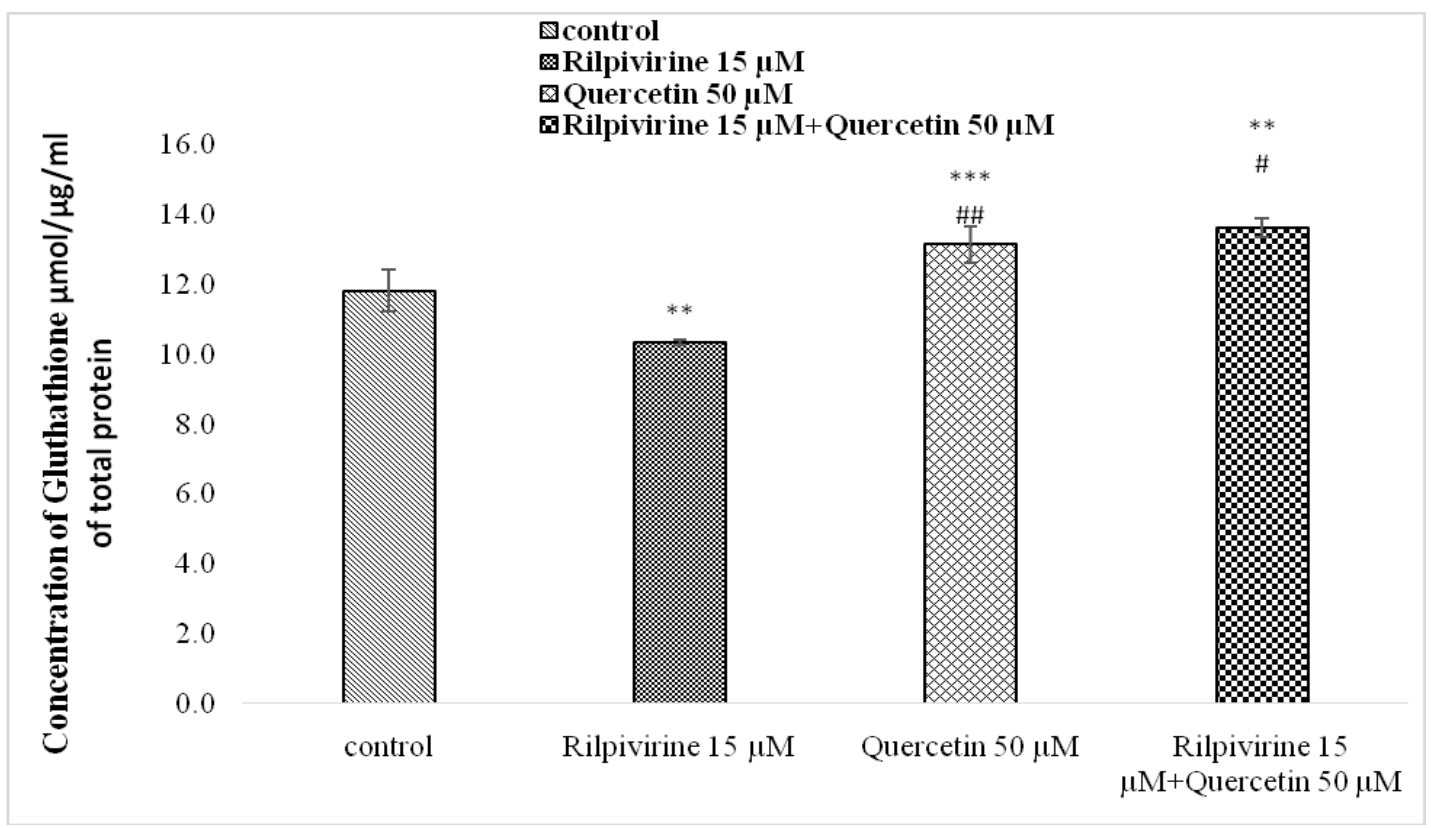

Fig V (A-D) Bar charts showing the effect of rilpivirine and quercetin individually and then in combination on the release of oxidative markers by human adipocytes in culture.

Human pre-adipocytes were differentiated in the presence of indicated concentrations of drugs ( $15 \mu \mathrm{M}$ rilpivirine and $50 \mu \mathrm{M}$ quercetin). Values represent concentrations of oxidative markers in the cell lysates for the 8 th day of culture. Statistical analysis was performed using a one-way ANOVA and are presented as means of \pm S.D from triplicate set of experiments expressed relative to values from untreated control cells; ${ }^{*} \mathrm{p}<0.05{ }^{*} \mathrm{p} p<0.01$ compared to the control. \# represents significance difference within the treatment groups. 
The data presented in figure V A show a small, but significant reduction in catalase activity in adipose cells treated with rilpivirine alone $(\mathrm{p}<0.01)$ as compared to control. In contrast, either quercetin or quercetin in combination with rilpivirine had do significant effect on catalase activity compared to control. The results in figure V B show a significant decrease $(\mathrm{p}<0.01)$ in SOD activity in adipose cells treated with rilpivirine alone. Adipose cells treated with quercetin alone showed a small, but significant $(\mathrm{p}<0.05)$ increase in the SOD activity. When rilpivirine was combined with quercetin they induced a small, but significant $(\mathrm{p}<0.05)$ increase in SOD activity compared to adipose cells treated with rilpivirine alone. Figure V C shows the levels of nitric oxide in adipose cells. The results show that rilpivirine alone can elicit a marked increase $(\mathrm{p}<0.05)$ in the nitric oxide levels as compared to control untreated cells. In contrast, nitric oxide levels were significantly $(\mathrm{p}<0.01)$ reduced in adipose cells treated with quercetin alone. However, when rilpivirine was combined with quercetin there was a small, but significant increase in nitric oxide levels $(\mathrm{p}<0.05)$. The results presented in figure V D show a significant decrease $(\mathrm{p}<0.01)$ in glutathione activity for adipose cells treated with rilpivirine alone. Quercetin alone and in combination with ripivirine evoked a marked but, significant $(\mathrm{p}<0.001)$ elevation in GSH activity as compared to control.

\section{DISCUSSION}

Over the last decade, the introduction of antiretroviral therapy (HAART) has dramatically decreased morbidity and mortality rates among HIV-infected patients [22]. However, multiple morphological and metabolic abnormalities including subcutaneous adipose tissue wasting (lipo-atrophy), central adipose tissue accumulation (lipo-hypertrophy), severe dyslipidemia and abnormalities of glucose metabolism have been observed among HAART-treated patients with well-controlled HIV. Previously reported data on anti-retroviral drug therapy have demonstrated serious metabolic consequences [23]. Although the new anti-retroviral drugs are not free of metabolic abnormalities, studies determining the effects on metabolic indices and body composition are limited. With this preview in mind, the present study investigated any detrimental effects of rilpivirine, a newly developed HIV drug, on human adipocytes as a model of inflammation, either alone or in combination with quercetin, a known plant-derived antioxidant. Firstly, the two drugs were investigated for any toxic effect on triglyceride accumulation and second, their ability to induce inflammation and oxidative stress.

In order to assess the cyto-toxicity of cells, human subcutaneous adipose cells were treated with either rilpivirine or quercetin alone and in combination and cell viability was assessed on day 3 and day 15 of culture. The results show that rilpivirine at lower concentrations $(10 \mu \mathrm{M}$ and $15 \mu \mathrm{M})$ and quercetin at $50 \mu \mathrm{M}$ did not alter the 
viability rate of the cells. However, at a concentration of $20 \mu \mathrm{M}$, rilpivirine induced a small, but significant $(\mathrm{p}<0.05)$ decrease in the cell viability (cell death). A combination of rilpivirine and quercetin at concentrations of $20 \mu \mathrm{M}$ and $50 \mu \mathrm{M}$, respectively, also elicited a significant $(\mathrm{p}<0.05)$ decrease in adipocyte viability. In the next series of experiments, human subcutaneous adipose cells were treated with either rilpivirine or quercetin alone and then in combination for a period of 15 days to determine triglyceride accumulation. Previous clinical trials conducted to measure the blood triglycerides, total cholesterol, low- and high-density lipoprotein in patients treated with rilpivirine showed substantial increases in these parameters compared to other NNRTIs belonging to the same class of antiretroviral drugs [24-26]. These findings were also confirmed by other studies [27]. The results from the present study show substantial reduction in the accumulation of triglyceride levels for adipose cells treated with either rilpivirine or quercetin alone and in combination, as evidenced by morphological pictures and quantitative measurement of triglycerides on the 5th, 8th, 11th and 15th day of the differentiation process. As compared to control, an altered lipid accumulation was observed in a dose- and timedependent manner. Higher concentration of rilpivirine $(20 \mu \mathrm{M})$ alone or in combination with quercetin severely inhibited triglyceride accumulation during adipocyte differentiation. Based on these results, a median drug concentration of $15 \mu \mathrm{M}$ of rilpivirine and differentiation for a period of 8 days were chosen for further experiments.

Alterations in inflammatory status of adipose tissue gives rise to a growing recognition that obesity represents a state of chronic low-level inflammation. In the present study, human subcutaneous adipose cells were treated with either $15 \mu \mathrm{M}$ rilpivirine or $50 \mu \mathrm{M}$ quercetin individually and in combination for a period of 8 days. The results show that rilpivirine can induce a marked reduction in the levels of adiponectin. Levels of other inflammatory markers such as resistin and IL-8 were increased as compared to the untreated cells. No significant change in leptin was observed on treatment of adipose cells with rilpivirine alone and its level was almost comparable to control. However, a significant reduction in inflammatory markers was observed for adipose cells treated with quercetin alone, thereby, revealing its ant-inflammatory properties. Combination of the two drugs markedly reduced the inflammatory makers.

As mentioned earlier, treatment with antiretroviral therapy may often result in biochemical and physiological changes including oxidative damage. This may result from either the presence of free radicals or the absence of antioxidants. So far, sufficient evidence to substantiate the role of free radicals in mediating oxidative injury is not present. Data from a series of previously conducted studies have reported an increase in oxidative stress, in 
addition to the persistent redox imbalance associated with HIV-1 infection manifested by an increase in oxidants and a decrease in antioxidant serum levels [28-30]. In order to reveal the oxidative status of the adipose cells treated with $15 \mu \mathrm{M}$ of rilpivirine or $50 \mu \mathrm{M}$ of quercetin either alone or in combination, a number of biomarkers of oxidative stress were measured quantitatively in this study. The generated oxidative profile in this study revealed a significant decrease in the levels of anti-oxidant enzymes like superoxide dismutase, catalase and glutathione in adipose cells treated with rilpivirine. The results also show a significant increase in the nitric oxide levels indicating its role in mitochondrial dysfunction. This reduction in the anti-oxidant species may possibly be attributed to the production of more oxidized metabolites deriving from the interaction between ROS and infected cell biomolecules [31-36]. It may further be explained and supported by several biochemical mechanisms, such as mitochondrial interference following treatment with ART [31, 35-36] and activation of the P450 hepatic system [32]. Both insufficient intake or mal-absorption of nutrients which are common attributes of HIV patients, may further worsen these conditions [37, 38].

In hope to lessen the side effects of antiretroviral therapy like lipo-dystrophy syndrome, rilpivirine at a concentration of $15 \mu \mathrm{M}$ was supplemented with $50 \mu \mathrm{M}$ of an anti-oxidant, quercetin. The results of this study show that quercetin supplementation significantly ameliorated rilpivirine- induced oxidative stress. It was able to prevent injury caused by free radicals by scavenging of ROS, activate antioxidant enzymes and mitigate the oxidative stress caused by nitric oxide. The overall increase in the levels of anti-oxidant enzymes when supplemented with quercetin may be attributed to its much reported anti-oxidative and direct radical scavenging properties. Quercetin, thus, seems to be a powerful flavonoid for protecting the body against reactive oxygen species, produced during the normal oxygen metabolism or are induced by exogenous damage [39, 40].

To summarize, soon after the introduction of the highly active antiretroviral therapy for the treatment of HIV infection, body shape changes and metabolic abnormalities were increasingly observed. Alongside, a tremendous expansion in the interest and use of traditional medicines worldwide, to combat such abnormalities has been on a rise. However, policies regarding the regulation of the production and use of herbs and other complimentary medicines and their quality, safety, efficacy and scientific evidence in relation to health claims is lacking. The present paper attempts to suspect pathophysiological relationships and the therapeutic consequences arising due to the antiretroviral drug, rilpivirine. This information may assist physicians in identifying individual risk factors and choosing the type of antiretroviral therapy that may minimize the associated metabolic changes without loss of virologic suppression. 
Present data show that rilpivirine, at a concentration of $15 \mu \mathrm{M}$, displays lipodystrophic effects by elucidating an increase in the inflammatory markers and suppressing the anti-oxidant enzymes. However, in vitro studies using cells in culture have obvious limitations with respect to their relevance to the treatment of patients. It should be taken into account that the overall alterations in lipid metabolism in HIV-1- infected patients are thought to be caused not by a single drug, but by the complex interactions of a given drug with other drug components of antiretroviral treatment regimens, as well as underlying HIV-1-infection-related alterations. Thus, further studies assessing lipid abnormalities in patients treated with rilpivirine as a component of a drug cocktail will be necessary to confirm a metabolic-friendly effect of this drug to identify potential lipid alterations. The study also, for the first time, reports co-administration of rilpivirine and quercetin. The results demonstrate the potential abilities of quercetin to attenuate oxidative damage and decrease inflammatory markers. However, the study warrants further mechanistic and clinical research studies employing protein and gene expression adipogenic markers to develop quercetin as a proven natural and effective agent for the ART-induced prevention or treatment of metabolic disturbances. It is also worth considering that lipodystrophy and overall alterations of lipid metabolism in HIV-1-infected patients analyzed to date are caused by complex interactions of a given drug with other cART components, as well as with underlying HIV-1 infection-related events.

\section{CONFLICT OF INTEREST}

The authors declare no conflict of interests with respect to the present paper.

\section{REFERENCES}

1. Bailey, A. C. and Fisher, M. (2008). Current use of antiretroviral treatment. British Medical Bulletin, 87(1) : $175-192$.

2. Palella,F.J., Delaney,K.M., Moorman,A.C., Loveless,M.O., Fuhrer,J., Satten, G.A., Aschman, D.J. and Holmberg, S.D. (1998). Declining Morbidity and Mortality among Patients with Advanced Human Immunodeficiency Virus Infection. New England Journal of Medicine. Massachusetts Medical Society, 338(13) : 853-860.

3. Montessori,V., Press, N., Harris, M., Akagi,L. and Montaner, J. (2004). Adverse effects of antiretroviral therapy for HIV infection. Canadian Medical Association Journal. 170(2) : 229-238.

4. Slaven, E. M., Lopez, F., Weintraub, S.L,. Mena, J.C. and Mallon, W.K. (2003). The AIDS patient with abdominal pain: a new challenge for the emergency physician. Emergency Medicine Clinics of North America, 21(4) : 987-1015.

5. Rasmussen, L. D., Kessel.L., Molander, L.D., Pedersen, C., Gerstoft, J., Kronborg, G. and Obel, N. (2011). Risk of Cataract Surgery in HIV-Infected Individuals: A Danish Nationwide Population-Based Cohort Study. Clinical Infectious Diseases, 53(11) : 1156-1163 
6. Liu, B., Zhang, L., Guo, R., Su, J., Li, L. and Si, Y. (2012). Anti-infective treatment in HIV-infected patients during perioperative period. AIDS Research and Therapy, $9: 36$.

7. Grulich, A. E., Van Leeuwen, M.T., Falster, M.O. and Vajdic, C.M. (2007). Incidence of cancers in people with HIV/AIDS compared with immunosuppressed transplant recipients: a meta-analysis. The Lancet, 370(9581) : 59-67.

8. World Health Organization (2003). Nutrient requirements for people living with HIV / AIDS: a report of a technical consultation', WHO Technical Consultation on Nutrient Requirements for People living with HIV/AIDS,pp.13-15.Available http://www.who.int/nutrition/publications/Content_nutrient_requirements.pdf.

9. World Health Organization [WHO] (1989a). In vitro screening of traditional medicines for anti-HIV activity: memorandum from a WHO meeting. Bull. World Health Organization. 67 613-618.

10. World Health Organization [WHO] (1989b). Report of a WHO Informal Consultation on Traditional Medicine and AIDS: In Vitro Screening for Anti-HIV Activity, Geneva, 6-8 February 1989. Geneva: World Health Organization.

11. Vermani, K. and Garg, S. (2002). Herbal medicines for sexually transmitted diseases and AIDS. Journal of Ethnopharmacology, 80(1) : 49-66.

12. Short, R.V. (2006). New ways of preventing HIV infection: thinking simply, simply thinking. Philosophical transactions of the Royal Society of London. Biological sciences, 361(1469) : 811-820.

13. Abbot, N. C. and Ernst, E. (1997). Patients' Opinions about Complementary Medicine. Complementary Medicine Research, 4(3) : 164-168.

14. Fairfield, K.M., Eisenberg, D.M., Davis, R,B., Libman, H. and Phillips, R.S. (1998). Patterns of use, expenditures, and perceived efficacy of complementary and alternative therapies in hiv-infected patients. Archives of Internal Medicine, 158(20) : 2257-2264.

15. Sparber, A., Wootton, J.C., Bauer, L., Curt, G., Eisenberg, D., Levin, T. and Steinberg, S.M. (2000). Use of Complementary Medicine by Adult Patients Participating in HIV/AIDS Clinical Trials. The Journal of Alternative and Complementary Medicine, 6(5) : 415-422.

16.Gore-Felton,C.,Vosvick,M., Power,R., Koopman, C., Ashton, E., Bachmann, M.H., Israelski, D. and Spiegel, D. (2003). Alternative Therapies: A Common Practice among Men and Women Living with HIV. Journal of the Association of Nurses in AIDS Care, 14(3) : 17-27.

17. Standish, L. J., Greene, K.B., Bain, S., Reeves, C., Sanders, F., Wines, R.C.M., Turet, P., Kim, J.G. and Calabrese,C. (2001).Alternative medicine use in HIV-positive men and women: Demographics, utilization patterns and health status. AIDS Care, 13(2) : 197-208.

18. Duggan, J., Peterson, W.S., Schutz, M., Khuder, S. and Charkraborty, J. (2001). Use of Complementary and Alternative Therapies in HIV-Infected Patients. AIDS Patient Care and STDs, 15(3) : 159-167.

19. Schrijvers, R., Desimmie, B. A. and Debyser, Z. (2011). Rilpivirine: a step forward in tailored HIV treatment. The Lancet, 378(9787) : 201-203.

20. Sultana, B. and Anwar, F. (2008). Flavonols (kaempeferol, quercetin, myricetin) contents of selected fruits, vegetables and medicinal plants. Food Chemistry, 108(3) : 879-884.

21. Salvamani, S., Gunasekaran, B., Shaharuddin, A.N., Ahmad, S.A. and Shukor, Y. (2014). Antiartherosclerotic effects of plant flavonoids. BioMed research international, Article I.D. 480258.

22. Detels, R. Muñoz, A., McFarlane, G., Kingsley, L.A., Margolick, J.B., Giorgi, J., Schrager, L.K. and Phair, J.P. (1998). Effectiveness of potent antiretroviral therapy on time to aids and death in men with known hiv 
infection duration., JAMA, 280(17) : 1497-1503.

23. Bacchetti, P., Gripshover, B., Grunfeld, C. et al. (2005). Fat distribution in men with HIV infection. Journal of Acquired Immune Deficiency Syndromes, 40(2): 121-131.

24. Cohen, C. J., Andrade-Villanueva, J., Clotet, B., Fourie, J., Johnson, M.A., Ruxrungtham, K., Wu, H., Zorrilla, C., Crauwels, H., Rimsky, L.T., Vanveggel, S. and Boven, K. (2011). Rilpivirine versus efavirenz with two background nucleoside or nucleotide reverse transcriptase inhibitors in treatment-naive adults infected with HIV-1 (THRIVE): a phase 3, randomised, non-inferiority trial. The Lancet, 378(9787): 229-237.

25. Molina, J. M., Cahn, P., Grinsztejn, B., Lazzarin, A., Mills, A., Saag, M., Supparatpinyo, K., Walmsley, S., Crauwels, H., Rimsky, L.T., Vanveggel, S. and Boven, K. (2011). Rilpivirine versus efavirenz with tenofovir and emtricitabine in treatment-naive adults infected with HIV-1 (ECHO): A phase 3 randomised double-blind active-controlled trial. The Lancet, 378(9787) : 238-246.

26. Nelson, M. R., Elion, R.A., Cohen. C.J., Mills, A., Hodder, S.L., Segal-Maurer, S., Bloch, M., Garner, W., Guyer, B., Williams, S., Chuck, S., Vanveggel, S., Deckx, H. and Stevens, M. (2013). Rilpivirine Versus Efavirenz in HIV-1-Infected Subjects Receiving Emtricitabine/Tenofovir DF: Pooled 96-Week Data from ECHO and THRIVE Studies. HIV Clinical Trials, 14(3) : 81-91.

27. Wilkin, A., Pozniak, A.L., Morales-Ramirez, J., Lupo, S.H., Santoscoy, M., Grinsztejn, B., Ruxrungtham, K., Rimsky, L.T., Vanveggel, S. and Boven, K. (2012). Long-term efficacy, safety, and tolerability of rilpivirine (RPV, TMC278) in HIV type 1-infected antiretroviral-naive patients: week 192 results from a phase IIb randomized trial. AIDS research and human retroviruses, 28(5) : 437-446.

28. Masiá, M., Padilla, S., Bernal, E., Almenar, M.V., Molina, J., Hernández, I., Graells, M.L. and Gutiérrez, F. (2007). Influence of Antiretroviral Therapy on Oxidative Stress and Cardiovascular Risk: A Prospective CrossSectional Study in HIV-Infected Patients. Clinical Therapeutics, 29(7) : 1448-1455.

29.Mandas,A., Iorio,E.L.,Congiu,M.G CinziaBalestrieri,C., Mereu,A., Cau,D., Dessì,S. and Nicoletta Curreli, N. (2009).Oxidative imbalance in HIV-1 infected patients treated with antiretroviral therapy. Journal of Biomedicine and Biotechnology, Article ID 749575.

30. Deavall, D. G., Martin, E.A., Horner, J.M. and Roberts, R. (2012). Drug-induced oxidative stress and toxicity. Journal of Toxicology, Article ID 645460.

31. de la Asunción, J.G., del Olmo, M.L., Sastre, J., Millán, A., Pellín, A., Pallardó, F.V. and Viña, J. (1998). AZT treatment induces molecular and ultrastructural oxidative damage to muscle mitochondria. Prevention by antioxidant vitamins. Journal of Clinical Investigation, 102(1) : 4-9.

32. Kumar, G. N., Dykstra, J., Roberts, E.M., Jayanti, V.K., Hickman, D., Uchic, J., Yao, Y., Surber, B., Thomas, S. and Granneman, G.R. (1999). Potent Inhibition of the Cytochrome P-450 3A-Mediated Human Liver Microsomal Metabolism of a Novel HIV Protease Inhibitor by Ritonavir: A Positive Drug-Drug Interaction. Drug Metabolism and Disposition, 27(8) : 902-908.

33. Hulgan, T., Morrow, J., D'Aquila, R.T., Raffanti, S., Morgan, M., Rebeiro, P. and Haas, D.W. (2003).Oxidant Stress Is Increased during Treatment of Human Immunodeficiency Virus Infection. Clinical Infectious Diseases, 37(12):1711-1717.

34. Lewis, W. (2003). Mitochondrial dysfunction and nucleoside reverse transcriptase inhibitor therapy: experimental clarifications and persistent clinical questions. Antiviral Research, 58(3): 189-197.

35. Cossarizza, A. and Moyle, G. (2004). Antiretroviral nucleoside and nucleotide analogues and mitochondria. Aids, 18(2) : 137-151.

36. Day, B. J. and Lewis, W. (2004). Oxidative stress in NRTI-induced toxicity: Evidence from clinical experience and experiments in vitro and in vivo. Cardiovascular Toxicology, 4(3) : 207-216. 
37. Tang, A. M., Lanzillotti, J., Hendricks, K., Gerrior, J., Ghosh, M., Woods, M. and Wanke, C. (2005). Micronutrients: Current issues for HIV care providers. Aids, 19(9):847-861.

38. Drain, P. K., Kupka, R., Mugusi, F. and Fawzi W.W. (2007). Micronutrients in HIV-positive persons receiving highly active antiretroviral therapy', The American Journal of Clinical Nutrition, 85(2) : 333-345.

39.Groot, D. H. (1994). Reactive oxygen species in tissue injury. Hepatogastroenterology, 41(4) : 328-332.

40. Grace, P. A. (1994). Ischaemia-reperfusion injury. The British Journal of Surgery, 81(5):637-647. 